\title{
Dynamics of Electric Field Induced Deformations in Homeotropic Bent-Core Nematic Layers
}

\author{
M. BuCZKOWSKA* AND J. GRACZYKOWSKI \\ Institute of Physics, Lodz University of Technology, Wólczańska 219, 90-924 Łódź, Poland \\ (Received October 16, 2019; revised version December 3, 2019; in final form December 5, 2019)
}

\begin{abstract}
Dynamics of deformations induced by dc electric field in homeotropically aligned layers containing a flexoelectric nematic liquid crystal composed of bent-core molecules was studied numerically. The respond times, characterizing the development of deformations after switching on the external voltage, and the decay times, describing the relaxation of deformation after switching off the voltage, were calculated for various values of flexoelectric coefficients. The results were compared with analogous behavior occurring in typical calamitic nematics. It was found that the unique properties of the bent-core nematic do not cause significant difference in the dynamics of deformations.
\end{abstract}

DOI: 10.12693/APhysPolA.137.390

PACS/topics: flexoelectricity, bent-core nematic, director deformations, director dynamics

\section{Introduction}

Nematic liquid crystals composed of molecules with bent core exhibit some unique features compared to conventional liquid crystals [1]. In particular, the ratios between their elastic constants are expressed by the inequalities $k_{11}>k_{33}>k_{22}$ which differ from typical relations found in nematics composed of calamitic (rod-like) molecules [2]. The bent shape of molecules gives rise to strong flexoelectric properties $[3,4]$. The dielectric anisotropy is usually negative [5]. The aim of the present work was to check whether these properties influence the dynamics of deformations induced by external electric field in plane-parallel nematic layers. For this purpose, the homeotropic layer containing the bent-core nematic material was modeled numerically. The deformations, occurring after application of external field and relaxation after switching off the field, were simulated. The time variation of optical transmission of the system composed of the nematic layer placed between crossed polarizers was calculated. Three characteristic times defined in Fig. 1, usually used as dynamic parameters describing the rise as well as the decay of deformation, were determined. They were calculated as functions of bias voltage and of flexoelectric coefficients.

\section{Geometry and parameters of the system}

The nematic layer of thickness $d=10 \mu \mathrm{m}$ confined between two plates parallel to the $x y$ plane of the Cartesian coordinate system and placed at $z= \pm d / 2$, was taken into account. The easy axes made the angle $\theta_{0}=1^{\circ}$ with the $z$ axis which ensured nearly homeotropic boundary

\footnotetext{
* corresponding author; e-mail: mariola.buczkowska@p.lodz.pl
}
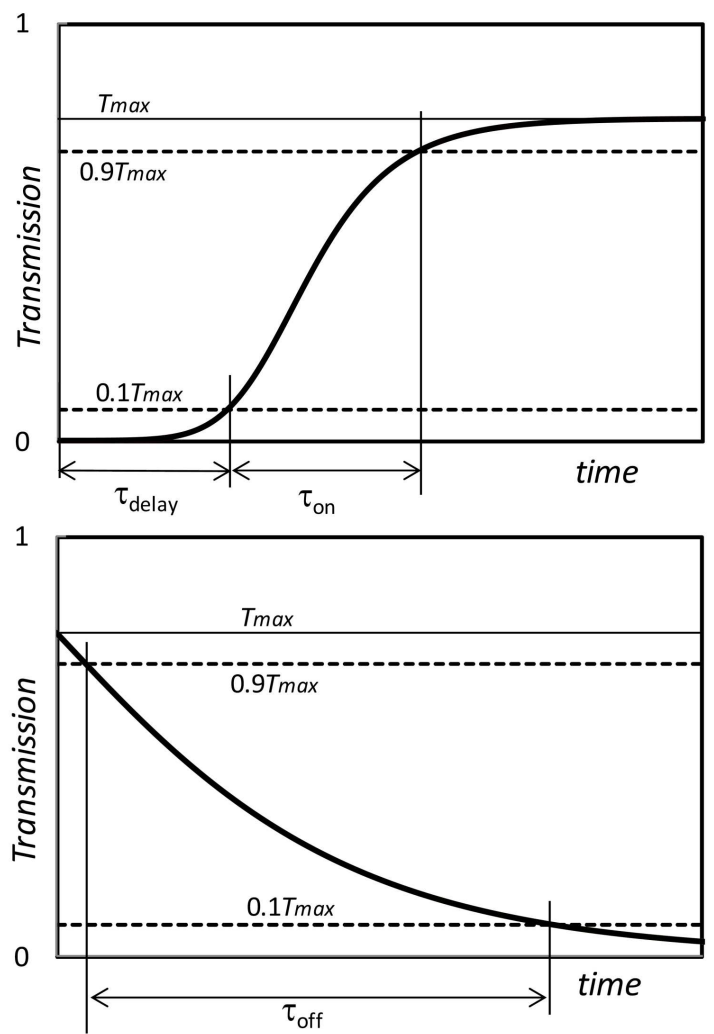

Fig. 1. Definitions of characteristic times describing the dynamics of deformation of the nematic layer.

conditions. The director $\boldsymbol{n}$ was parallel to the $x z$ plane. Its orientation was described by the angle $\theta(z, t)$, measured between $\boldsymbol{n}$ and the $z$-axis. The limiting plates played the role of electrodes. The lower electrode was earthed. The deformation arose after application of the voltage step of amplitude $U$. The decay of distortion occurred when the zero voltage was applied to the state of saturated static deformation. The model 
bent-core nematic was characterized by the elastic constants $k_{11}=6.0 \times 10^{-12} \mathrm{~N}, k_{22}=1.2 \times 10^{-12} \mathrm{~N}$, and $k_{33}=3.5 \times 10^{-12} \mathrm{~N}[6]$. Negative dielectric anisotropy, $\Delta \varepsilon=-4$ was adopted, and the value of $\varepsilon_{\perp}$ was 16 . The flexoelectric properties were expressed by the sum of flexoelectric coefficients $e=e_{11}+e_{33}$, which was varied between 0 and $50 \mathrm{pC} / \mathrm{m}$. The rotational viscosity $\gamma_{1}=0.1093 \mathrm{~N} \mathrm{~s} \mathrm{~m}^{-2}$ and the surface viscosity $\kappa=2.6 \times 10^{-8} \mathrm{~N} \mathrm{~s} \mathrm{~m}^{-1}$ determined the rheological properties $[7,8]$. The backflow effect was neglected. The interactions between nematic and boundary surfaces were determined by the anchoring strength parameter $W=10^{-4} \mathrm{~J} \mathrm{~m}^{-2}$. In order to calculate the optical transmission, the refractive indices $n_{o}=1.520$ and $n_{e}=1.672$ and a wavelength of $\lambda=589 \mathrm{~nm}$ were adopted. The transport of ionic charge of concentration $N_{0}=10^{18} \mathrm{~m}^{-3}$ was also taken into account. It was described in terms of a weak electrolyte model which was used in earlier paper $[9,10]$. It is presented in Appendix.

\section{Method}

The system was considered as one-dimensional. Its time evolution was given by the set of equations which described balance of elastic, viscous, dielectric, and flexoelectric torques acting in the bulk. The boundary conditions were expressed by two equations of balance of elastic, viscous, flexoelectric, and anchoring torques for the boundaries [9]. The electrical properties of the layer were described by the Poisson equation, two equations of continuity of the ion fluxes which govern the transport of ions in the bulk and four equations describing the electrode processes which play the role of boundary conditions. The quasi-blocking properties of the electrode contacts were assumed. Detailed forms of all equations are given in Appendix, where the numerical method is also described.

\section{Results and discussion}

The computations revealed the time variation of director configurations described by the angle $\theta(z, t)$, as it is exemplified in Fig. 2. The varying director distributions allowed to calculate the time dependences of the optical transmission of the layer placed between crossed polarizers, $T(t)$. Optical transmission of the undistorted homeotropic layer was practically zero. Small deformations of the director distribution induced by bias voltage led to initial increase of transmission. Stronger deformations resulted in oscillations of transmission, which were beyond our interest. The widths of voltage ranges were limited to the lowest values which corresponded to transmission varying between zero and $100 \%$. Figure 3 shows exemplary time variations of transmission calculated for arising of deformation after application of the voltage as well as for decay of deformation after switching off the voltage. The dependences of this kind allowed to
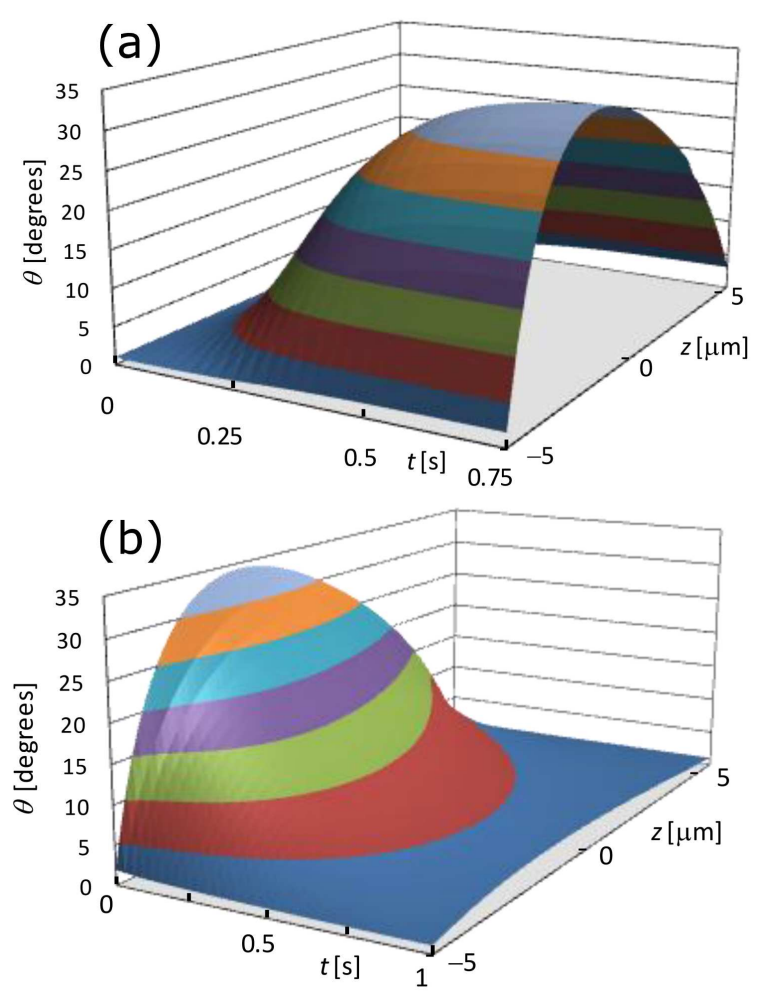

Fig. 2. Example of the director orientation angle $\theta$ as a function of time and coordinate for arising (a) and decaying (b) of deformation; $U=1.8 \mathrm{~V}, e=50 \mathrm{pC} / \mathrm{m}$.
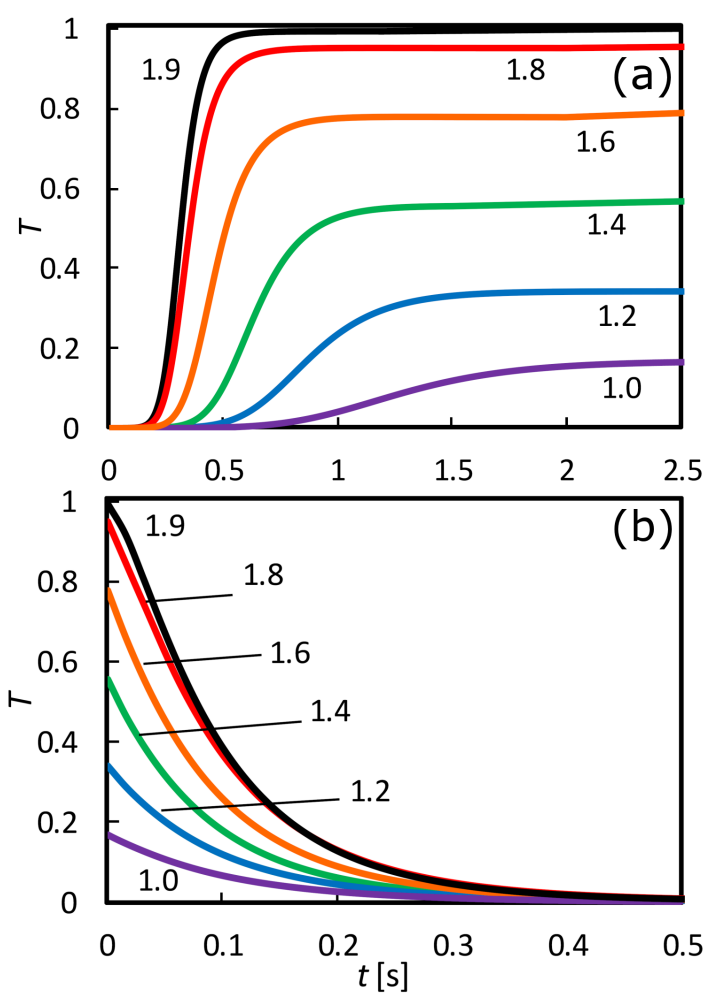

Fig. 3. Optical transmission as a function of time after application of voltage step (a) and after switching off the voltage (b) for a bent-core nematic; values of voltage (in $\mathrm{V})$ are given at the curves; $e=50 \mathrm{pC} / \mathrm{m}$. 

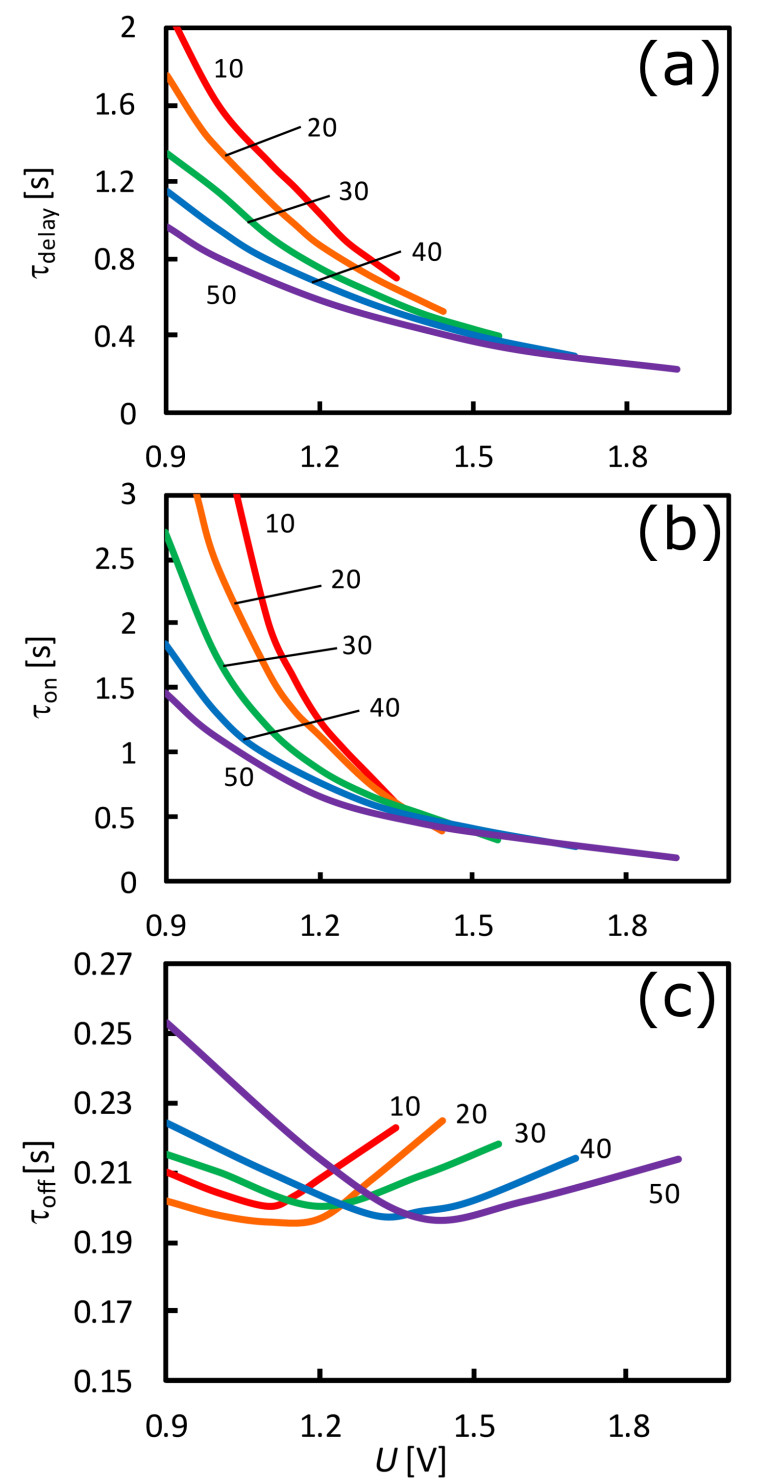

Fig. 4. The delay time (a), the rise time (b) and the decay time (c) as functions of voltage for a bent-core nematic; the values of flexoelectric coefficients $e$ (in $\mathrm{pC} / \mathrm{m}$ ) are given at the curves.

determine the characteristic times which describe the dynamics of deformations. The times are plotted in Fig. 4 for various flexoelectric coefficients $e$. It is evident that the delay times and the rise times had similar values. They decreased monotonically with voltage. They also decreased significantly with flexoelectric coefficient. The decay times were much shorter. Their voltage dependence was more complex. There was some voltage for which $\tau_{\text {off }}$ reached a minimum. At small voltages, the stronger flexoelectric properties led to longer decay time. Reverse dependence was found for high voltages.

The results presented above can be compared with corresponding dependences occurring in calamitic nematics. For this purpose, additional computations were performed for typical nematic material characterized

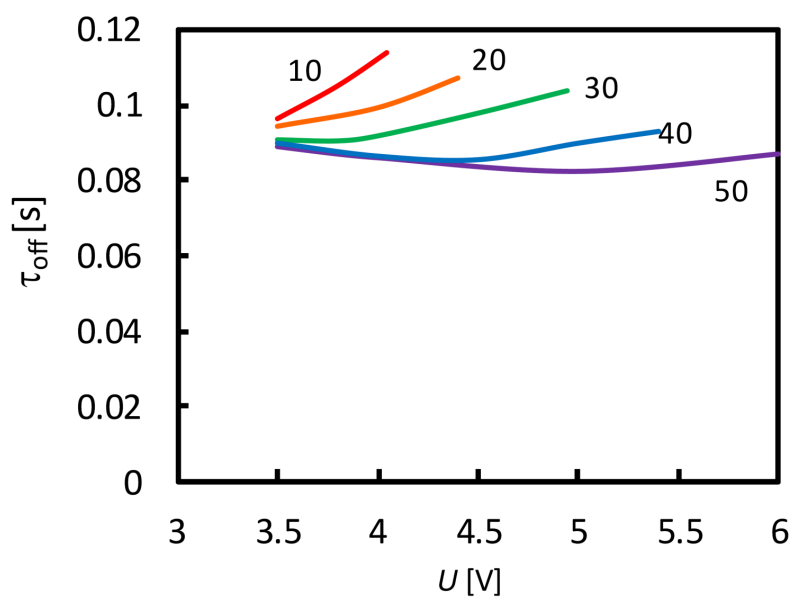

Fig. 5. The decay time as function of voltage for typical calamitic nematic; the values of flexoelectric coefficients $e$ (in $\mathrm{pC} / \mathrm{m}$ ) are given at the curves.

by $k_{11}=6.0 \times 10^{-12} \mathrm{~N} \quad k_{22}=3.8 \times 10^{-12} \mathrm{~N}$, $k_{33}=7.5 \times 10^{-12} \mathrm{~N}$, and $\Delta \varepsilon=-0.7$. It was found that $\tau_{\text {delay }}$ and $\tau_{\text {on }}$ obtained for typical calamitic nematic were qualitatively identical with those obtained for the bent-core nematic. On the contrary, the decay time $\tau_{\text {off }}$ slightly increased with voltage and decreased with flexoelectric coefficients (Fig. 5).

The dynamics of deformations occurring in nematic layers is described sometimes by means of the time constants calculated from linear part of the time dependence of logarithm of the optical retardation of the layer. However in the present case such dependences were not linear, so the time constants could not be determined.

Dynamics of deformations is governed by the torques acting on the system. Arising of deformations occurs due to destabilizing dielectric torque acting on the whole layer. The flexoelectric torque is destabilizing in vicinity of the negative electrode and stabilizing in the neighborhood of the positive electrode. This asymmetry is due to influence of ionic space charge which contributes to electric field distribution in the layer [11]. The flexoelectric torques acting on the boundary surfaces have negligible influence because they are overwhelmed by strong surface anchoring strength. The decay of deformations is driven mainly by the surface interactions restoring the homeotropic structure, however, the elastic constant also affects this process which is illustrated by the difference between the decay times calculated for bent-core and calamitic nematics (Figs. 4c and 5, respectively). This effect can be explained if the torque equation (A1) is taken into account. The elastic torque depends on ratio of elastic constants $k_{b}=k_{33} / k_{11}$ which is significantly larger for calamitic nematic $\left(k_{b}=1.25\right)$ than for the bent core material $\left(k_{b}=0.58\right)$. The deformation of the homeotropic layer is influenced mainly by the $k_{b}$ ratio due to the term $k_{b} \cos ^{2} \theta$ of the torque. The twice larger value of $k_{b}$ for calamitic substance makes it more rigid which results in twice shorter decay time. 


\section{Appendix A: The set of equations and the outline of the numerical method $[9,10]$}

The time evolution of the considered deformation was described by the following set of equations:

- equation of balance of elastic, viscous, dielectric, and flexoelectric torques for the bulk

$$
\begin{aligned}
& \frac{1}{2}\left(k_{b}-1\right) \sin 2 \theta(\zeta, t)\left(\frac{\partial \theta(\zeta, t)}{\partial \zeta}\right)^{2} \\
& -\left(k_{b} \cos ^{2} \theta(\zeta, t)+\sin ^{2} \theta(\zeta, t)\right) \frac{\partial^{2} \theta(\zeta, t)}{\partial \zeta^{2}} \\
& +\frac{1}{2} \frac{\varepsilon_{0} \Delta \varepsilon}{k_{11}} \sin 2 \theta(\zeta, t)\left(\frac{\partial V(\zeta, t)}{\partial \zeta}\right)^{2} \\
& +\frac{1}{2} \frac{e_{11}+e_{33}}{k_{11}} \sin 2 \theta(\zeta, t)\left(\frac{\partial^{2} V(\zeta, t)}{\partial \zeta^{2}}\right)= \\
& \quad-\frac{\gamma_{1} d^{2}}{k_{11}} \frac{\partial \theta(\zeta, t)}{\partial t},
\end{aligned}
$$

where $\zeta=z / d$ is a reduced coordinate;

- two equations of balance of elastic, viscous, flexoelectric and anchoring torques for the boundaries which play the role of boundary conditions, for $\zeta=-1 / 2$ :

$$
\begin{aligned}
& -\left.\frac{1}{2} \frac{e_{11}+e_{33}}{k_{11}} \sin 2 \theta(-1 / 2, t) \frac{\partial V(\zeta, t)}{\partial \zeta}\right|_{-1 / 2, t}(\mathrm{~A} 2) \\
& +\left.\left[k_{b} \cos ^{2} \theta(-1 / 2, t)+\sin ^{2} \theta(-1 / 2, t)\right] \frac{\partial \theta(\zeta, t)}{\partial \zeta}\right|_{-1 / 2, t} \\
& \quad+\frac{1}{2} \omega \sin 2\left(\theta(-1 / 2, t)-\theta_{0}\right)=-\kappa \frac{d}{k_{11}} \frac{\partial \theta(-1 / 2, t)}{\partial t},
\end{aligned}
$$

and for $\zeta=1 / 2$ :

$$
\begin{gathered}
\left.\frac{1}{2} \frac{e_{11}+e_{33}}{k_{11}} \sin 2 \theta(1 / 2, t) \frac{\partial V(\zeta, t)}{\partial \zeta}\right|_{1 / 2, t} \\
-\left.\left[k_{b} \cos ^{2} \theta(1 / 2, t)+\sin ^{2} \theta(1 / 2, t)\right] \frac{\partial \theta(\zeta, t)}{\partial \zeta}\right|_{1 / 2, t} \\
\quad+\frac{1}{2} \omega \sin 2\left(\theta(1 / 2, t)-\theta_{0}\right)=-\kappa \frac{d}{k_{11}} \frac{\partial \theta(1 / 2, t)}{\partial t}
\end{gathered}
$$

where $\omega=W d / k_{11}$;
- the Poisson equation

$$
\begin{aligned}
\varepsilon_{0}\left[\varepsilon_{\perp}+\Delta \varepsilon \cos ^{2} \theta(\zeta, t)\right] \frac{\partial^{2} V(\zeta, t)}{\partial \zeta^{2}} \\
-\varepsilon_{0} \Delta \varepsilon \sin 2 \theta(\zeta, t) \frac{\partial V(\zeta, t)}{\partial \zeta} \frac{\partial \theta(\zeta, t)}{\partial \zeta} \\
+\left(e_{11}+e_{33}\right) \cos 2 \theta(\zeta, t)\left(\frac{\partial \theta(\zeta, t)}{\partial \zeta}\right)^{2} \\
+\frac{1}{2}\left(e_{11}+e_{33}\right) \sin 2 \theta(\zeta, t) \frac{\partial^{2} \theta(\zeta, t)}{\partial \zeta^{2}} \\
+q\left[N^{+}(\zeta, t)-N^{-}(\zeta, t)\right] d^{2}=0
\end{aligned}
$$

with the boundary conditions $V(-1 / 2, t)=0$ and $V(1 / 2)=U$ for the rise of the deformation and $V(-1 / 2, t)=V(1 / 2)=0$ for the decay;

- two equations of continuity of the ion fluxes which govern the transport of ions in the bulk based on the weak electrolyte model

$$
\frac{\partial N^{+}(\zeta, t)}{\partial t} d=\left[\beta-\alpha N^{+}(\zeta, t) N^{-}(\zeta, t)\right] d-\frac{\partial J_{z}^{+}(\zeta, t)}{\partial \zeta},
$$

$$
\frac{\partial N^{-}(\zeta, t)}{\partial t} d=\left[\beta-\alpha N^{+}(\zeta, t) N^{-}(\zeta, t)\right] d-\frac{\partial J_{z}^{-}(\zeta, t)}{\partial \zeta}
$$

where $\beta$ and $\alpha$ are the generation and recombination constants, respectively [12], and

$$
\begin{aligned}
& J_{z}^{+}=-\frac{1}{d}\left(\mu_{z z}^{+} N^{+}(\zeta, t) \frac{\partial V(\zeta, t)}{\partial \zeta}+D_{z z}^{+} \frac{\partial N^{+}(\zeta, t)}{\partial \zeta}\right) \\
& J_{z}^{-}=\frac{1}{d}\left(\mu_{z z}^{-} N^{-}(\zeta, t) \frac{\partial V(\zeta, t)}{\partial \zeta}-D_{z z}^{-} \frac{\partial N^{-}(\zeta, t)}{\partial \zeta}\right)
\end{aligned}
$$

denote the fluxes of ions of given sign. The $z$-components of the mobility and diffusion coefficients are given by $\mu_{z z}^{ \pm}=\mu_{\perp}^{ \pm}+\Delta \mu^{ \pm} \cos ^{2} \theta$ and $D_{z z}^{ \pm}=D_{\perp}^{ \pm}+\Delta D^{ \pm} \cos ^{2} \theta$, respectively, where $\Delta \mu^{ \pm}=\mu_{\|}^{ \pm}-\mu_{\perp}^{ \pm}$and $\Delta D^{ \pm}=D_{\|}^{ \pm}-D_{\perp}^{ \pm}$ denote the anisotropies of both quantities;

- four equations describing the electrode processes which play the role of boundary conditions for $\zeta=-1 / 2$ :

$$
\begin{aligned}
& \frac{\partial N^{+}(-1 / 2, t)}{\partial t} L=-N^{+}(-1 / 2, t) K_{r} \exp \left(\Delta \phi(-1 / 2, t) / k_{\mathrm{B}} T\right)+N_{0} K_{r} \exp \left(-\Delta \phi(-1 / 2, t) / k_{B} T\right) \\
& \quad+\left(\left.\mu_{z z}^{+} N^{+}(-1 / 2, t) \frac{\partial V(\zeta, t)}{\partial \zeta}\right|_{(-1 / 2, t)}+\left.D_{z z}^{+} \frac{\partial N^{+}(\zeta, t)}{\partial \zeta}\right|_{(-1 / 2, t)}\right) \frac{1}{d} \\
& \frac{\partial N^{-}(-1 / 2, t)}{\partial t} L=-N^{-}(-1 / 2, t) K_{r} \exp \left(-\Delta \phi(-1 / 2, t) / k_{\mathrm{B}} T\right)+N_{0} K_{r} \exp \left(\Delta \phi(-1 / 2, t) / k_{\mathrm{B}} T\right) \\
& \quad-\left(\left.\mu_{z z}^{-} N^{-}(-1 / 2, t) \frac{\partial V(\zeta, t)}{\partial \zeta}\right|_{(-1 / 2, t)}-\left.D_{z z}^{-} \frac{\partial N^{-}(\zeta, t)}{\partial \zeta}\right|_{(-1 / 2, t)}\right) \frac{1}{d}
\end{aligned}
$$


and for $\zeta=1 / 2$ :

$$
\begin{aligned}
& \frac{\partial N^{+}(1 / 2, t)}{\partial t} L=-N^{+}(1 / 2, t) K_{r} \exp \left(-\Delta \phi(1 / 2, t) / k_{\mathrm{B}} T\right)+N_{0} K_{r} \exp \left(\Delta \phi(1 / 2, t) / k_{\mathrm{B}} T\right) \\
& \quad-\left(\left.\mu_{z z}^{+} N^{+}(1 / 2, t) \frac{\partial V(\zeta, t)}{\partial \zeta}\right|_{(1 / 2, t)}+\left.D_{z z}^{+} \frac{\partial N^{+}(\zeta, t)}{\partial \zeta}\right|_{(1 / 2, t)}\right) \frac{1}{d} \\
& \frac{\partial N^{-}(1 / 2, t)}{\partial t} L=-N^{-}(1 / 2, t) K_{r} \exp \left(\Delta \phi(1 / 2, t) / k_{\mathrm{B}} T\right)+N_{0} K_{r} \exp \left(-\Delta \phi(1 / 2, t) / k_{B} T\right) \\
& \quad+\left(\left.\mu_{z z}^{-} N^{-}(1 / 2, t) \frac{\partial V(\zeta, t)}{\partial \zeta}\right|_{(1 / 2, t)}-\left.D_{z z}^{-} \frac{\partial N^{-}(\zeta, t)}{\partial \zeta}\right|_{(1 / 2, t)}\right) \frac{1}{d}
\end{aligned}
$$

where the parameter $K_{r}$ describes the quasi-blocking properties of the electrode contacts. The parameter $L$ is the thickness of the sub-electrode region, of the order of several molecular lengths, where the electrode reactions occur. The quantities $\Delta \varphi( \pm 1 / 2, t)=|E( \pm 1 / 2, t)| q L$ are the electric field induced changes of the energy barriers between the nematic and the electrodes.

The initial conditions for the orientation angle in the case of arising of deformation were given by $\theta(\zeta, 0)=$ $\theta_{0}=1^{\circ}$. The initial conditions for the potential were imposed by $V(\zeta, 0)=U \zeta+U / 2$ and those for the ion concentrations by $N_{0}^{ \pm}(\zeta, 0)=N_{0}$. In the case of decay of deformation, the initial conditions were determined by the variables describing the static state of the layer subjected to the chosen voltage, $\theta(\zeta, 0), V(\zeta, 0)$, and $N^{ \pm}(\zeta, 0)$.

The above set of equations was solved numerically. The variables $\theta(\zeta, t), V(\zeta, t)$, and $N^{ \pm}(\zeta, t)$ were approximated by their discrete values $\theta_{i j}, V_{i j}$, and $N_{i j}^{ \pm}$defined in the nodes of the $M \times N$ regular lattice. The indices $i=1, \ldots, M$ determined the positions along the $z$ coordinate while $j=1, \ldots, N$ determined the discrete moments of time. The difference equations obtained from Eqs. (A4)-(A12) formed the sets described by tridiagonal matrices and were solved by means of the sweep method. In order to solve the torque Eqs. (A1)-(A3), the actual relaxation of director in a nematic sample, occurring when the torques of different origin do not balance, was simulated. Namely, the total torque $\boldsymbol{\Gamma}_{i j}$ acting on the director in the node $i j$ of the lattice was calculated. In a real sample, this torque rotates the director. The new director orientation was found by adding a small vector $\Delta \boldsymbol{n}=c \boldsymbol{\Gamma} \times \boldsymbol{n}$, where $c$ is a suitably chosen constant ensuring that $|\Delta \boldsymbol{n}| \ll 1$. The absolute value of new director was then normalized to 1 and the corresponding orientation angle $\theta_{i j}$ was calculated. As a result, the value of $\boldsymbol{\Gamma}_{i j}$ was gradually decreased. All the procedures mentioned above were repeated sufficiently many times for the whole lattice. This yielded the sets of values $\theta_{i j}, V_{i j}$, and $N_{i j}^{ \pm}$which approximated well the functions $\theta(\zeta, t)$, $V(\zeta, t)$, and $N^{ \pm}(\zeta, t)$ satisfying Eqs. (A1)-(A12).
The results allowed us to calculate the time dependent phase difference between the ordinary and extraordinary rays passing through a layer placed between crossed polarisers, expressed by the formula

$$
\begin{aligned}
& \Delta \Phi(t)=\frac{2 \pi d}{\lambda} \\
& \times\left(\int_{-1 / 2}^{1 / 2} \frac{n_{e} n_{o}}{\left(n_{e}^{2} \cos ^{2} \theta(\zeta, t)+n_{o} \sin ^{2} \theta(\zeta, t)\right)^{1 / 2} \mathrm{~d} \zeta-n_{o}}\right) .
\end{aligned}
$$

as well as the optical transmission of the system, $T=\sin ^{2}(\Delta \Phi / 2)$. Finally, the times $\tau_{\text {delay }}, \tau_{\text {on }}$, and $\tau_{\text {off }}$ were determined.

\section{References}

[1] A. Jákli, Liq. Cryst. Rev. 1, 65 (2013).

[2] S. Kaur, Liq. Cryst. 43, 2277 (2016).

[3] J. Harden, B. Mbanga, N. Éber, K. Fodor-Csorba, S. Sprunt, J.T. Gleeson, A. Jákli, Phys. Rev. Lett. 97, 157802 (2006).

[4] Á. Buka, N. Éber, K. Fodor-Csorba, A. Jákli, P. Salamon, Phase Transit. 85, 872 (2012).

[5] P. Salamon, N. Éber, Á. Buka, J.T. Gleeson, S. Sprunt, A. Jákli, Phys. Rev. E 81, 031711 (2010).

[6] H.F. Gleeson, S. Kaur, V. Görtz, A. Belaissaoui, S. Cowling, J.W. Goodby, ChemPhysChem 15, 1251 (2014).

[7] C. Gähwiller, Phys. Lett. A 36, 311 (1971).

[8] A.G. Petrov, A.Th. Ionescu, C. Versace, N. Scaramuzza, Liq. Cryst. 19, 169 (1995).

[9] G. Derfel, M. Buczkowska, Liq. Cryst. 40, 272 (2013).

[10] G. Derfel, J. Mol. Liq. 144, 59 (2009).

[11] M. Buczkowska, G. Derfel, Opto-Electron. Rev. 19 56 (2011).

[12] H. de Vleeschouwer, A. Verschueren, F. Bougriona, R. Van Asselt, E. Alexander, S. Vermael, K. Neyts, H. Pauwels, Jpn J. Appl. Phys. 40, 3272 (2001). 